\title{
Research Article \\ Boundary Layer Equations and Lie Group Analysis of a Sisko Fluid
}

\author{
Gözde Sarı, ${ }^{1,2}$ Mehmet Pakdemirli, ${ }^{1,2}$ \\ Tasawar Hayat, ${ }^{3,4}$ and Yiğit Aksoy ${ }^{1}$ \\ ${ }^{1}$ Department of Mechanical Engineering, Celal Bayar University, Muradiye, 45140 Manisa, Turkey \\ ${ }^{2}$ Applied Mathematics and Computation Center, Celal Bayar University, Muradiye, 45140 Manisa, Turkey \\ ${ }^{3}$ Department of Mathematics, Quaid-i-Azam University 45320, Islamabad 44000, Pakistan \\ ${ }^{4}$ Department of Mathematics, King Saud University, P.O. Box 2455, Riyadh 11451, Saudi Arabia
}

Correspondence should be addressed to Mehmet Pakdemirli, mpak@cbu.edu.tr

Received 27 September 2012; Accepted 5 December 2012

Academic Editor: Fazal M. Mahomed

Copyright (c) 2012 Gözde Sarı et al. This is an open access article distributed under the Creative Commons Attribution License, which permits unrestricted use, distribution, and reproduction in any medium, provided the original work is properly cited.

Boundary layer equations are derived for the Sisko fluid. Using Lie group theory, a symmetry analysis of the equations is performed. A partial differential system is transferred to an ordinary differential system via symmetries. Resulting equations are numerically solved. Effects of nonNewtonian parameters on the solutions are discussed.

\section{Introduction}

Due to the inadequacy of Newtonian fluid model which predicts a linear relationship between the shear stress and velocity gradient, many non-Newtonian fluid models were proposed to explain the complex behavior. Usually, the stress constitutive relations of such models inherit complexities which lead to highly nonlinear equations of motion with many terms. To simplify the extremely complex equations, one alternative is to use boundary layer theory which is known to effectively reduce the complexity of Navier-Stokes equations and reduce drastically the computational time. Since there are many non-Newtonian models and new models are being proposed continuously, boundary layer theory for each proposed model also appears in the literature. It is beyond the scope of this work to review vast literature on the boundary layers of non-Newtonian fluids. A limited work on the topic can be found in [1-19].

In this work, boundary layer theory is developed for the Sisko fluid, a non-Newtonian fluid model which combines the features of viscous and power law models. A complete 
symmetry analysis of the boundary layer equations is presented. Using one of the symmetries, the partial differential system is transformed into an ordinary differential system. The resulting ordinary differential system is numerically solved by a finite difference algorithm. Effect of non-Newtonian parameters on the velocity profiles is shown in the graphs.

As a non-Newtonian model, Sisko [20] proposed a new model which is later called sisko fluid. Some of the recent work on Sisko fluids is as follows: an analytical solution was presented using homotopy analysis method for the flow of a magnetohydrodynamic Sisko fluid through a porous medium [21]. Wire coating analysis was investigated by withdrawal from a bath of Sisko fluid [22]. The equation modeling thin film flow of a Sisko fluid on a moving belt qualitatively was analyzed and a series solution was found using homotopy analysis method [23]. Unsteady unidirectional flow of a Sisko fluid bounded by a suddenly moved plate was studied [24]. Unsteady flow of a Sisko fluid over a moving wall with suction or blowing was considered [25]. A numerical study for the unsteady flow of a magnetohydrodynamic Sisko fluid in annular pipe was presented [26]. Heat transfer was also incorporated into the annular pipe problem [27]. To the best of authors' knowledge, boundary layer analysis and symmetry reductions of Sisko fluids do not exist in the literature.

\section{Boundary Layer Equations}

The Cauchy stress tensor for Sisko fluid is

$$
\mathbf{T}=-p \mathbf{I}+\mathbf{S}
$$

where

$$
\begin{aligned}
& \mathbf{S}=\left[a+b\left|\sqrt{\frac{1}{2} \operatorname{tr}\left(\mathbf{A}_{1}^{2}\right)}\right|^{n-1}\right] \mathbf{A}_{1} \\
& \mathbf{A}_{1}=\mathbf{L}+\mathbf{L}^{T}, \quad \mathbf{L}=\operatorname{grad} \mathbf{V} .
\end{aligned}
$$

$\mathbf{V}$ is the velocity vector and $\mathbf{A}_{1}$ is the first Rivlin Ericksen tensor. $a$ and $b$ are the material constants. The model is a combination of viscous and power-law models. For $a=0$, the model exhibits power-law behavior whereas, for $b=0$, the flow is Newtonian. Steady-state, two-dimensional, incompressible equations of motion including mass conservation can be written as

$$
\begin{gathered}
\frac{\partial u^{*}}{\partial x^{*}}+\frac{\partial v^{*}}{\partial y^{*}}=0 \\
\rho\left(u^{*} \frac{\partial u^{*}}{\partial x^{*}}+v^{*} \frac{\partial u^{*}}{\partial y^{*}}\right)=-\frac{\partial p^{*}}{\partial x^{*}}+\frac{\partial S_{x x}}{\partial x^{*}}+\frac{\partial S_{x y}}{\partial y^{*}} \\
\rho\left(u^{*} \frac{\partial v^{*}}{\partial x^{*}}+v^{*} \frac{\partial v^{*}}{\partial y^{*}}\right)=-\frac{\partial p^{*}}{\partial y^{*}}+\frac{\partial S_{x y}}{\partial x^{*}}+\frac{\partial S_{y y}}{\partial y^{*}},
\end{gathered}
$$


where $x^{*}$ is the spatial coordinate along the surface, $y^{*}$ is vertical to it, $u^{*}$ and $v^{*}$ are the velocity components in the $x^{*}$ and $y^{*}$ coordinates. The shear stress components are inserted into the equations of motion and the usual boundary layer assumptions are made, that is, $x^{*} \sim O(1), y^{*} \sim O(\delta), u^{*} \sim O(1), v^{*} \sim O(\delta)$. The highest order terms are retained and the momentum equations become

$$
\begin{gathered}
\rho\left(u^{*} \frac{\partial u^{*}}{\partial x^{*}}+v^{*} \frac{\partial u^{*}}{\partial y^{*}}\right)=-\frac{\partial p^{*}}{\partial x^{*}}+a \frac{\partial^{2} u^{*}}{\partial y^{* 2}}+b n\left(\frac{\partial u^{*}}{\partial y^{*}}\right)^{n-1} \frac{\partial^{2} u^{*}}{\partial y^{* 2}} \\
0=\frac{\partial p^{*}}{\partial y^{*}}
\end{gathered}
$$

from which dependence of pressure on $y^{*}$ is eliminated. Dimensionless variables and parameters are defined as follows:

$$
x=\frac{x^{*}}{L}, \quad y=\frac{y^{*}}{L}, \quad u=\frac{u^{*}}{V}, \quad v=\frac{v^{*}}{V}, \quad p=\frac{p^{*}}{\rho V^{2}} \varepsilon_{1}=\frac{a}{\rho V L}, \quad \varepsilon_{2}=\frac{b n V^{n-2}}{\rho L^{n}},
$$

where $L$ is a characteristic length and $V$ is a reference velocity. Expressing the pressure in terms of outer velocity, the final dimensionless boundary layer equations become

$$
\begin{gathered}
\frac{\partial u}{\partial x}+\frac{\partial v}{\partial y}=0 \\
u \frac{\partial u}{\partial x}+v \frac{\partial u}{\partial y}=U \frac{d U}{d x}+\varepsilon_{1} \frac{\partial^{2} u}{\partial y^{2}}+\varepsilon_{2}\left(\frac{\partial u}{\partial y}\right)^{n-1} \frac{\partial^{2} u}{\partial y^{2}}
\end{gathered}
$$

The classical boundary conditions for the problem are

$$
u(x, 0)=0, \quad v(x, 0)=0, \quad u(x, \infty)=U(x) .
$$

For $\varepsilon_{2}=0$ or $n=1$, the equations reduce qualitatively to those of Newtonian fluid.

\section{Lie Group Theory and Symmetry Reductions}

Lie group Theory is employed in search of symmetries of the equations. Details of the theory can be found in $[28,29]$. The infinitesimal generator for the problem is

$$
X=\xi_{1}(x, y, u, v) \frac{\partial}{\partial x}+\xi_{2}(x, y, u, v) \frac{\partial}{\partial y}+\eta_{1}(x, y, u, v) \frac{\partial}{\partial u}+\eta_{2}(x, y, u, v) \frac{\partial}{\partial v}
$$

A straightforward and tedious calculation (see [28, 29] for details) yields

$$
\xi_{1}=3 a x+b, \quad \xi_{2}=a y+c(x), \quad \eta_{1}=a u, \quad \eta_{2}=c^{\prime}(x) u-a v .
$$


The classifying relation for the outer velocity is

$$
(3 a x+b) \frac{d}{d x}\left(U U^{\prime}\right)+a\left(U U^{\prime}\right)=0
$$

Symbolic packages developed to calculate symmetries fail to produce the above results due to the arbitrary outer velocity function, and hand calculation becomes inevitable for precise results. There are two finite parameter Lie group symmetries represented by parameters " $a$ " and " $b$," the former corresponding to scaling symmetry and the latter to translational symmetry in the $x$ coordinate. There is an additional infinite parameter Lie group symmetry represented by $c(x)$ function. For a general stress tensor which is an arbitrary function of the velocity gradient, the symmetries were calculated previously [18]. Our results for the special case of Sisko fluid confirm the previous calculations (see principal Lie Algebra presented in [18]).

Usually boundary conditions put much restriction on the symmetries which may lead to removal of all the symmetries. In our case, however, some of the symmetries remain stable after imposing the boundary conditions. For nonlinear equations, the generators should be applied to the boundaries and boundary conditions also [28]. Applying the generator to the boundary $y=0$ yields $c(x)=0$. Applying the generator to the boundary conditions does not impose further restrictions and hence the symmetries valid for the equations and boundary conditions reduce to

$$
\xi_{1}=3 a x+b, \quad \xi_{2}=a y, \quad \eta_{1}=a u, \quad \eta_{2}=-a v
$$

with the classifying relation (3.3) remaining unchanged.

Selecting parameter " $a$ " in the symmetries, the associated equations which define similarity variables are

$$
\frac{d x}{3 x}=\frac{d y}{y}=\frac{d u}{u}=\frac{d v}{-v}
$$

Solving the system yields the similarity variables

$$
\xi=\frac{y}{x^{1 / 3}}, \quad u=x^{1 / 3} f(\xi), \quad v=\frac{g(\xi)}{x^{1 / 3}}
$$

From (3.3) with $b=0, U(x)=x^{1 / 3}$. Substituting all into the boundary layer equations yields the ordinary differential system

$$
\begin{gathered}
f-\xi f^{\prime}+3 g^{\prime}=0 \\
f^{2}-\xi f f^{\prime}+3 g f^{\prime}=1+3 \varepsilon_{1} f^{\prime \prime}+3 \varepsilon_{2}\left(f^{\prime}\right)^{n-1} f^{\prime \prime} .
\end{gathered}
$$

The boundary conditions also transform to

$$
f(0)=0, \quad g(0)=0, \quad f(\infty)=1 .
$$




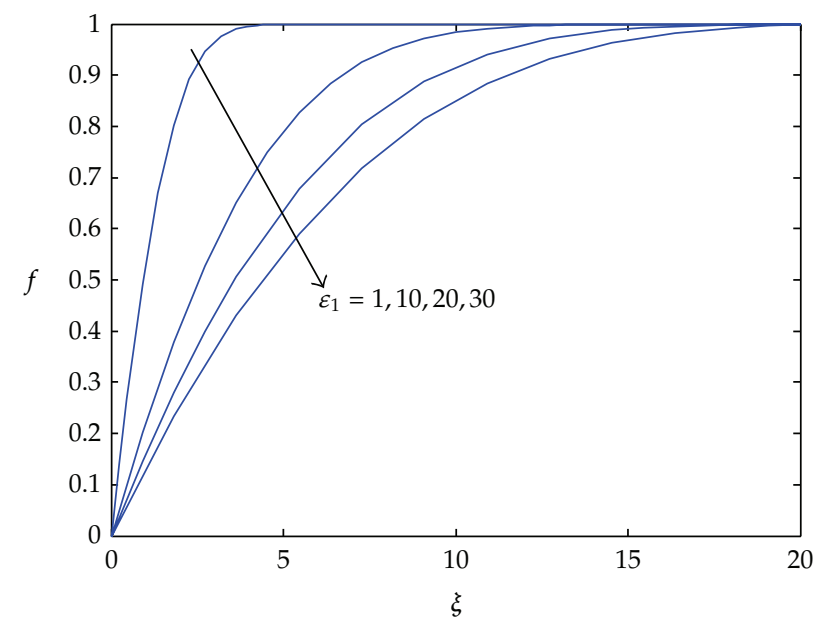

Figure 1: Effect of $\varepsilon_{1}$ parameter on the similarity function $f$ related to the $x$ component of velocity $\left(\varepsilon_{2}=1\right.$, $n=2)$.

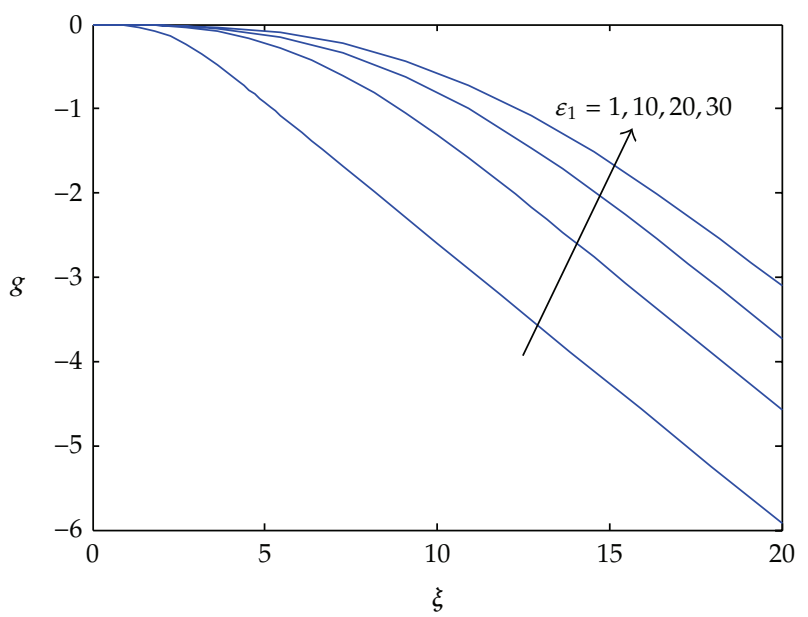

Figure 2: Effect of $\varepsilon_{1}$ parameter on the similarity function $g$ related to the $y$ component of velocity $\left(\varepsilon_{2}=1\right.$, $n=2)$.

\section{Numerical Results}

Equation (3.7) with the boundary conditions (3.8) are numerically integrated using a finite difference code. The finite difference code implements the three-stage Lobatto IIIA formula that provides continuous solutions of fourth order accuracy in the problem domain. Description of the numerical method is given by Shampine et al. [30] and Kierzenka and Shampine [31]. In Figure 1, $f$ function and in Figure 2, $g$ function related to the $x$ and $y$ components of the velocities are drawn for different $\varepsilon_{1}$ parameters. Boundary layer becomes thicker for an increase in $\varepsilon_{1}$.

There is an increase in $y$ component of velocity which is negative over the whole domain excluding the surface for an increase in $\varepsilon_{1}$ as can be seen from Figure 2. A similar trend is observed for parameter $\varepsilon_{2}$, although not as influential as in the previous case (see Figures 3 and 4). 


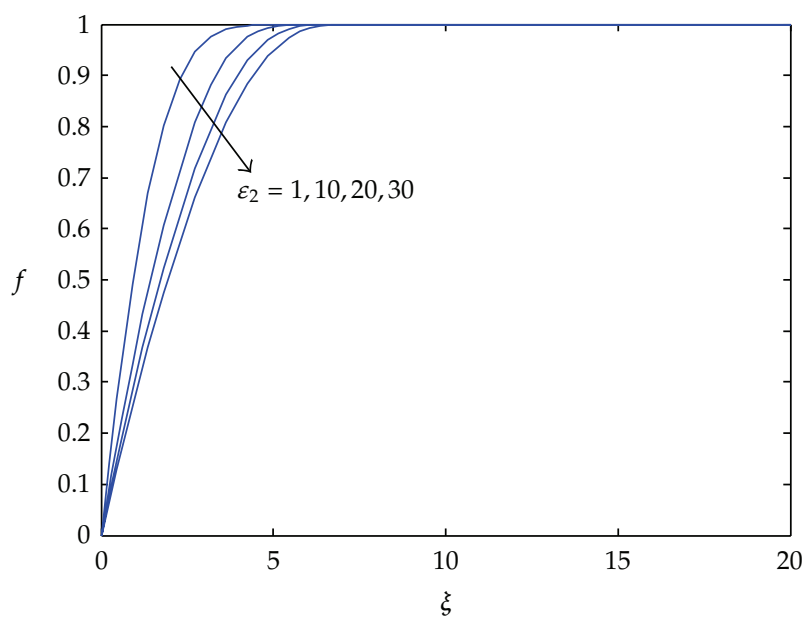

Figure 3: Effect of $\varepsilon_{2}$ parameter on the similarity function $f$ related to the $x$ component of velocity $\left(\varepsilon_{1}=1\right.$, $n=2)$.

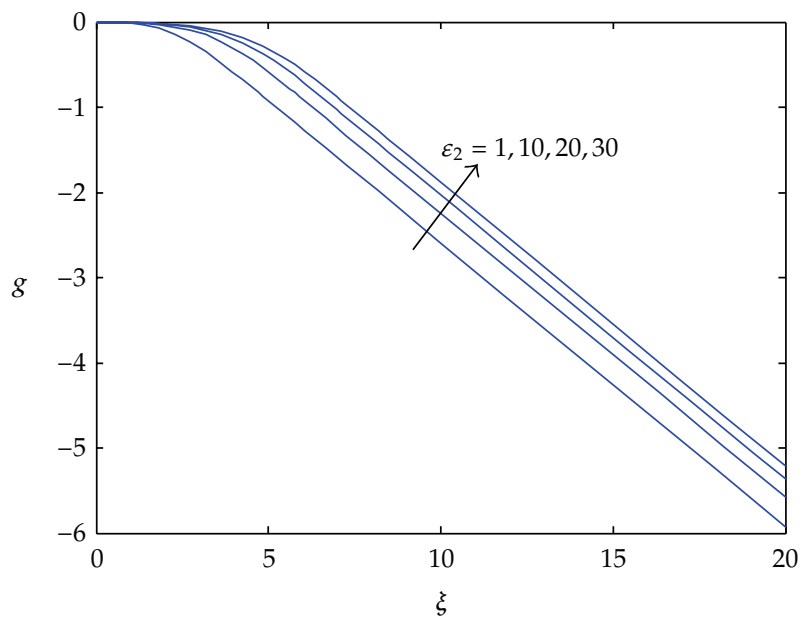

Figure 4: Effect of $\varepsilon_{2}$ parameter on the similarity function $g$ related to the $y$ component of velocity $\left(\varepsilon_{1}=1\right.$, $n=2)$.

On the contrary, a reverse effect is observed for power index $n$, that is, as $n$ increases, boundary layer becomes narrower eventually converging to a fixed thickness (see Figure 5). For the term related to $y$ component of velocity, higher values of power index $n$ cause a decrease which eventually converges to a solution as can be seen from Figure 6.

\section{Concluding Remarks}

Boundary layer equations of Sisko fluid derived for the first time. Lie group theory is applied to the equations. Equations admit two finite parameter Lie group transformations and an infinite parameter Lie group transformation. The infinite parameter Lie group transformation is not stable with respect to usual boundary layer conditions. Using the scaling symmetry 


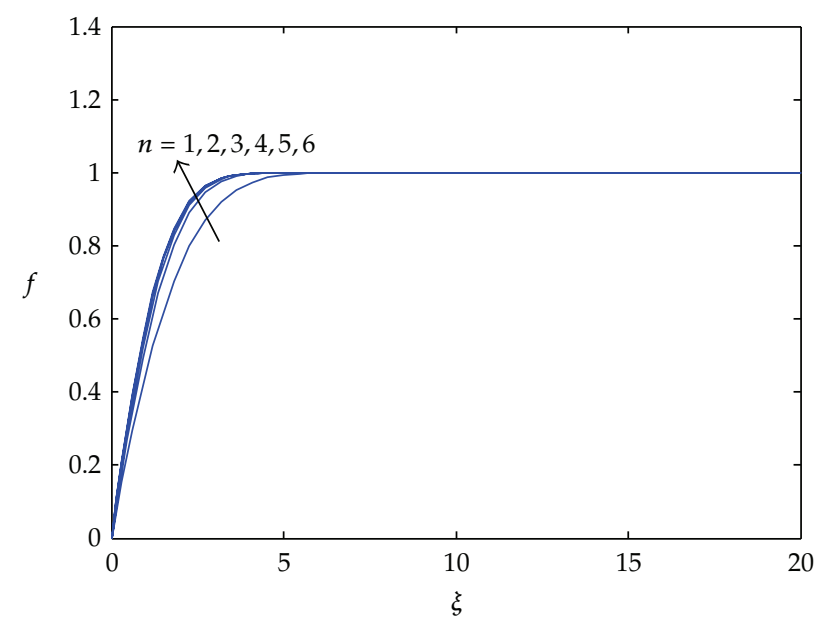

Figure 5: Effect of power index $n$ on the similarity function $f$ related to the $x$ component of velocity $\left(\varepsilon_{1}=1\right.$, $\left.\varepsilon_{2}=1\right)$.

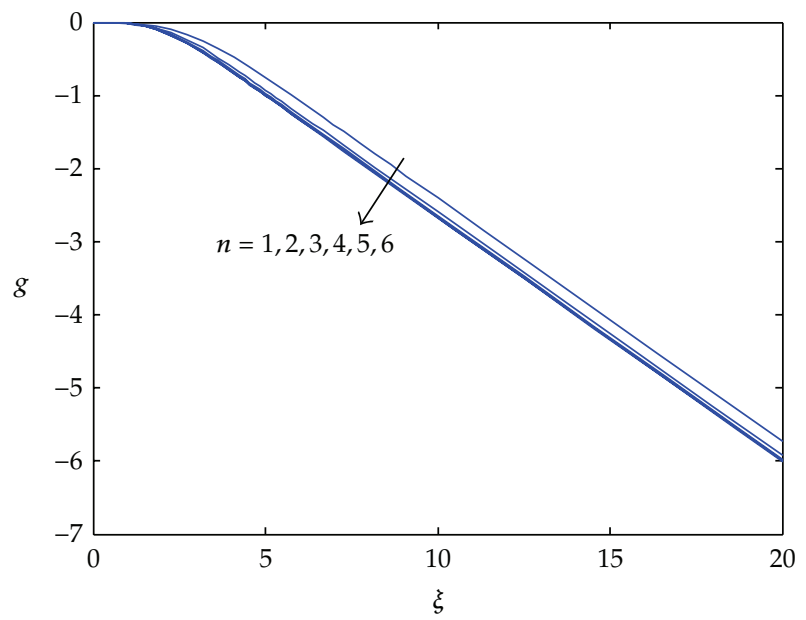

Figure 6: Effect of power index $n$ on the similarity function $g$ related to the $y$ component of velocity $\left(\varepsilon_{1}=1\right.$, $\left.\varepsilon_{2}=1\right)$.

which is one of the finite parameter transformations, the partial differential system is transferred into an ordinary differential system. Resulting ordinary differential system is solved numerically using a finite difference scheme. Effects of non-Newtonian parameters on the boundary layers are discussed in detail. It is found that an increase in the parameters $\varepsilon_{1}$ and $\varepsilon_{2}$ causes the boundary layer to thicken whereas a reverse effect is observed for power index $n$.

\section{Acknowledgments}

This work is completed during mutual short visits of T. Hayat to Turkey and M. Pakdemirli to Pakistan. Funding supports of TUBITAK of Turkey and HEC of Pakistan are highly appreciated. 


\section{References}

[1] A. Acrivos, M. J. Shahand, and E. E. Petersen, "Momentum and heat transfer in laminar boundary layer flows of non-Newtonian fluids past external surfaces," AIChE Journal, vol. 6, pp. 312-317, 1960.

[2] W. R. Schowalter, "The application of boundary-layer theory to power-law pseudoplastic fluids: similarity solutions," AIChE Journal, vol. 6, pp. 25-28, 1960.

[3] G. D. Bizzell and J. C. Slattery, "Non-newtonian boundary-layer flow," Chemical Engineering Science, vol. 17, no. 10, pp. 777-782, 1962.

[4] A. C. Srivastava, "The flow of a non-Newtonian liquid near a stagnation point," Zeitschrift für Angewandte Mathematik und Physik, vol. 9, no. 1, pp. 80-84, 1958.

[5] D. W. Beard and K. Walters, "Elastico-viscous boundary-layer flows," Proceedings of Cambridge Philosophical Society, vol. 60, pp. 667-674, 1964.

[6] J. Astin, R. S. Jones, and P. Lockyer, "Boundary layers in non-Newtonian fluids," Journal Mechanique, vol. 12, no. 3, pp. 527-539, 1973.

[7] K. R. Rajagopal, A. S. Gupta, and A. S. Wineman, “On a boundary layer theory for non-Newtonian fluids," International Journal of Engineering Science, vol. 18, no. 6, pp. 875-883, 1980.

[8] K. R. Rajagopal, A. S. Gupta, and T. Y. Na, "A note on the Falkner-Skan flows of a non-Newtonian fluid," International Journal of Non-Linear Mechanics, vol. 18, no. 4, pp. 313-320, 1983.

[9] V. K. Garg and K. R. Rajagopal, "Flow of a non-Newtonian fluid past a wedge," Acta Mechanica, vol. 88, no. 1-2, pp. 113-123, 1991.

[10] M. Massoudi and M. Ramezan, "Effect of injection or suction on the Falkner-Skan flows of second grade fluids," International Journal of Non-Linear Mechanics, vol. 24, no. 3, pp. 221-227, 1989.

[11] V. K. Garg and K. R. Rajagopal, "Stagnation point flow of a non-Newtonian fluid," Mechanics Research Communications, vol. 17, no. 6, pp. 415-421, 1990.

[12] M. Pakdemirli and E. S. Şuhubi, "Boundary layer theory for second order fluids," International Journal of Engineering Science, vol. 30, no. 4, pp. 523-532, 1992.

[13] M. Pakdemirli, "The boundary layer equations of third-grade fluids," International Journal of NonLinear Mechanics, vol. 27, no. 5, pp. 785-793, 1992.

[14] M. Pakdemirli, "Boundary layer flow of power-law fluids past arbitrary profiles," IMA Journal of Applied Mathematics, vol. 50, no. 2, pp. 133-148, 1993.

[15] R. K. Bhatnagar, G. Gupta, and K. R. Rajagopal, "Flow of an Oldroyd-B fluid due to a stretching sheet in the presence of a free stream velocity," International Journal of Non-Linear Mechanics, vol. 30, no. 3, pp. 391-405, 1995.

[16] T. Hagen and M. Renardy, "Boundary layer analysis of the Phan-Thien-Tanner and Giesekus model in high Weissenberg number flow," Journal of Non-Newtonian Fluid Mechanics, vol. 73, no. 1-2, pp. 181-189, 1997.

[17] M. Yürüsoy and M. Pakdemirli, "Symmetry reductions of unsteady three-dimensional boundary layers of some non-Newtonian fluids," International Journal of Engineering Science, vol. 35, no. 8, pp. 731-740, 1997.

[18] M. Yürüsoy and M. Pakdemirli, "Group classification of a non-Newtonian fluid model using classical approach and equivalence transformations," International Journal of Non-Linear Mechanics, vol. 34, no. 2, pp. 341-346, 1999.

[19] Y. Aksoy, M. Pakdemirli, and C. M. Khalique, "Boundary layer equations and stretching sheet solutions for the modified second grade fluid," International Journal of Engineering Science, vol. 45, no. 10, pp. 829-841, 2007.

[20] A. W. Sisko, "The flow of lubricating greases," Industrial and Engineering Chemistry, vol. 50, no. 12, pp. 1789-1792, 1958.

[21] M. Khan, Z. Abbas, and T. Hayat, "Analytic solution for flow of Sisko fluid through a porous medium," Transport in Porous Media, vol. 71, no. 1, pp. 23-37, 2008.

[22] M. Sajid and T. Hayat, "Wire coating analysis by withdrawal from a bath of Sisko fluid," Applied Mathematics and Computation, vol. 199, no. 1, pp. 13-22, 2008.

[23] F. T. Akyıldız, K. Vajravelu, R. N. Mohapatra, E. Sweet, and R. A. Van Gorder, "Implicit differential equation arising in the steady flow of a Sisko fluid," Applied Mathematics and Computation, vol. 210, no. 1, pp. 189-196, 2009.

[24] M. Molati, T. Hayat, and F. Mahomed, "Rayleigh problem for a MHD Sisko fluid," Nonlinear Analysis. Real World Applications, vol. 10, no. 6, pp. 3428-3434, 2009.

[25] T. Hayat, R. J. Moitsheki, and S. Abelman, "Stokes' first problem for Sisko fluid over a porous wall," Applied Mathematics and Computation, vol. 217, no. 2, pp. 622-628, 2010. 
[26] M. Khan, Q. Abbas, and K. Duru, "Magnetohydrodynamic flow of a Sisko fluid in annular pipe: a numerical study," International Journal for Numerical Methods in Fluids, vol. 62, no. 10, pp. 1169-1180, 2010.

[27] M. Khan, S. Munawar, and S. Abbasbandy, "Steady flow and heat transfer of a Sisko fluid in annular pipe," International Journal of Heat and Mass Transfer, vol. 53, no. 7-8, pp. 1290-1297, 2010.

[28] G. W. Bluman and S. Kumei, Symmetries and Differential Equations, vol. 81, Springer, New York, NY, USA, 1989.

[29] H. Stephani, Differential Equations: Their Solution Using Symmetries, Cambridge University Press, New York, NY, USA, 1989.

[30] L. F. Shampine, I. Gladwell, and S. Thompson, Solving ODEs with MATLAB, chapter 3, Cambridge University Press, Cambridge, UK, 2003.

[31] J. Kierzenka and L. F. Shampine, "A BVP solver based on residual control and the MATLAB PSE," Transactions on Mathematical Software, vol. 27, no. 3, pp. 299-316, 2001. 


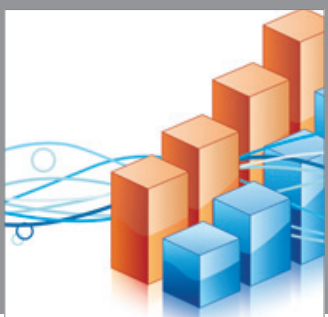

Advances in

Operations Research

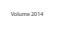

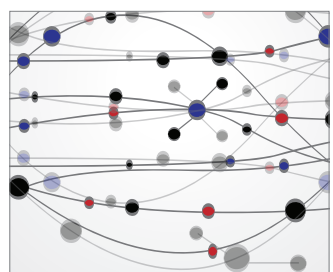

\section{The Scientific} World Journal
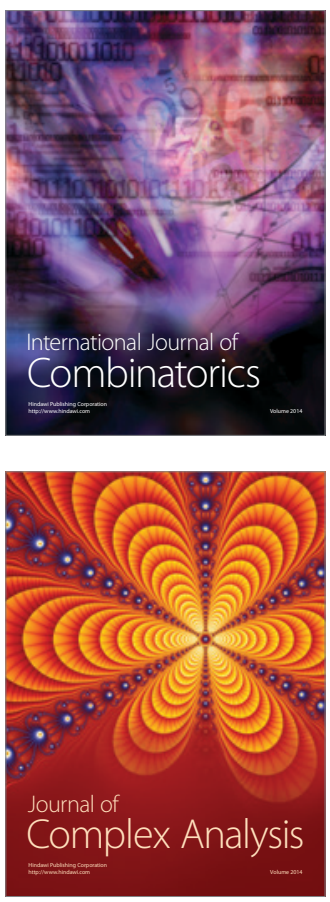

International Journal of

Mathematics and

Mathematical

Sciences
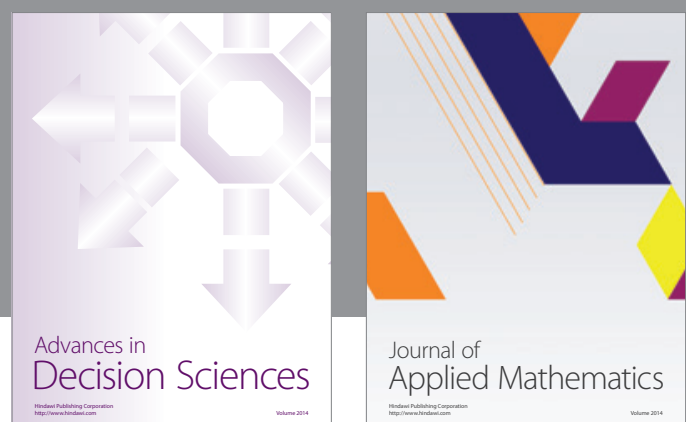

Journal of

Applied Mathematics
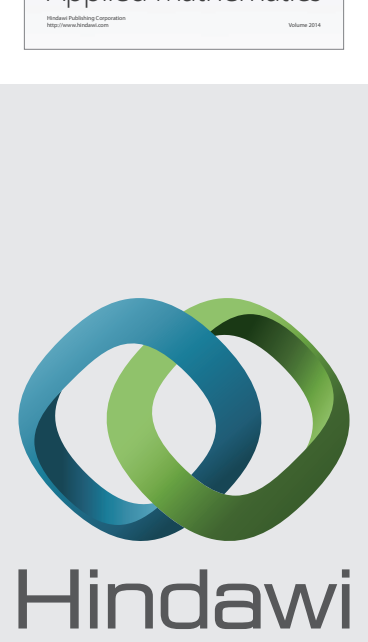

Submit your manuscripts at http://www.hindawi.com
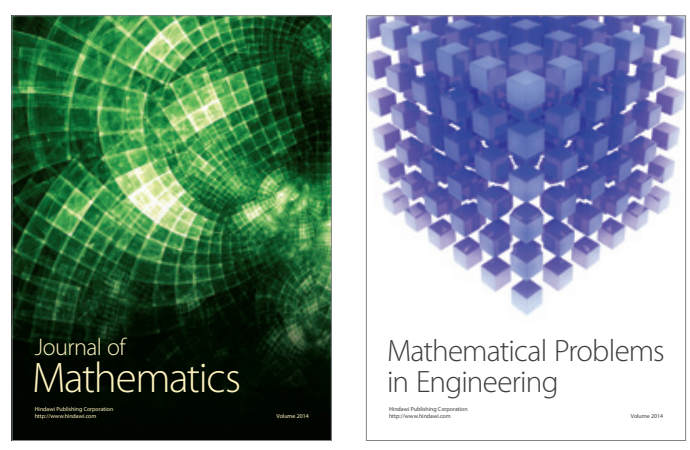

Mathematical Problems in Engineering
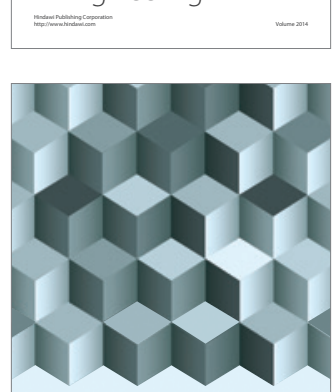

Journal of

Function Spaces
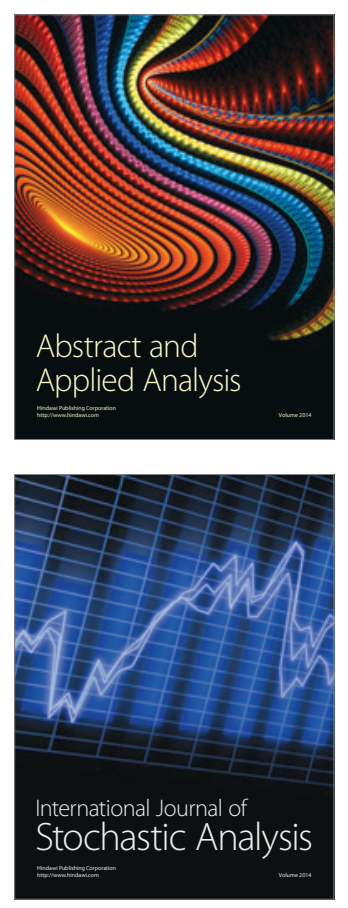

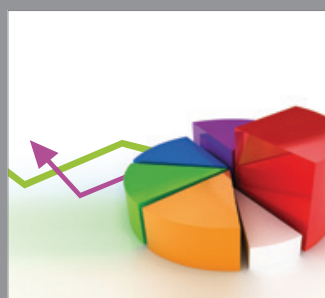

ournal of

Probability and Statistics

Promensencen
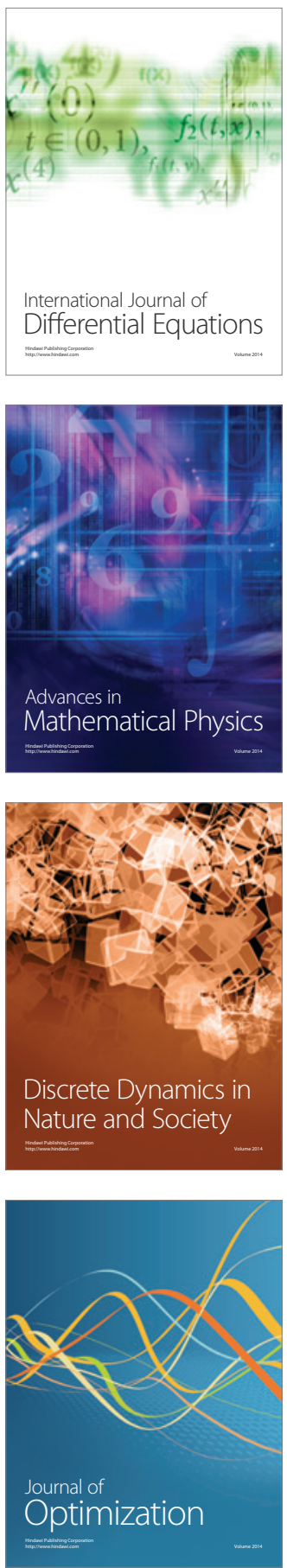